\title{
Fluoxetine plus cognitive behavioural therapy improves symptoms of major depressive disorder in adolescents
}

March J, Silva S, Petrycki S, et al. Fluoxetine, cognitive-behavioral therapy, and their combination for adolescents with depression: treatment for adolescents with depression study (TADS) randomized controlled trial. JAMA 2004;292:807-20.

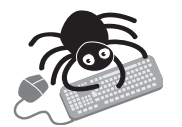

This article contains extra text on the EBMH website

Does fluoxetine plus cognitive behavioural therapy improve symptoms of depression in adolescents with major depressive disorder?

\section{METHODS}

$\square$

Design: Randomised controlled trial

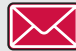

Allocation: Concealed.

Blinding: Single blind (assessors blinded to treatment).

Follow up period: 12 weeks.

Setting: Thirteen academic and community clinics, USA; spring 2000 to summer 2003

Patients: 439 adolescents with major depressive disorder (aged 12-17 years). Exclusions: non-English speaking; $I Q<80$; high risk of suicide; taking antidepressants or psychotropic drugs; diagnosis of bipolar, pervasive developmental or thought disorder; substance abuse; pregnant or refused to take birth control; or receiving psychotherapy.

D Intervention: Fluoxetine (10-40 mg/day) plus cognitive behavioural therapy (CBT); fluoxetine alone; CBT alone; or placebo. Participants receiving CBT took part in 15 one hour sessions with a therapist. Sessions included: psychoeducation about depression, goal setting, mood monitoring cognitive restructuring, social problem solving, and increasing pleasurable activities (week 1-6); tailored sessions to improve social skills (week 7-12). Parents also received two psychoeducation sessions without the adolescent present

Outcomes: Improvement in symptoms of depression (Children's Depression Rating Scale-Revised, Clinical Global Impressions improvement score), and reduction in suicidal thoughts (Suicidal Ideation Questionnaire-Junior High School Version).

Patient follow up: $80 \%$

\section{MAIN RESULTS}

At 12 weeks, fluoxetine plus CBT significantly improved symptoms of depression compared with placebo in adolescents with major depressive disorder ( $\mathrm{p}=0.00 \mathrm{l}$; see http://www.ebmentalhealth.com/ supplemental for table). Fluoxetine plus CBT was more effective at reducing symptoms of depression than fluoxetine alone or CBT alone (fluoxetine plus CBT $v$ fluoxetine, $\mathrm{p}=0.02$; fluoxetine plus CBT $v$ CBT, $p=0.001)$. There was no significant difference in reduction of depressive symptoms between fluoxetine alone or CBT alone compared with placebo (fluoxetine $v$ placebo, $\mathrm{p}=0.1$; CBT $v$ placebo,

For correspondence: John S March MD MPH, Department of Psychiatry, Duke University Medical Center, 718 Rutherford Street, Box 3527, Durham, NC 27710, USA; jsmarch@acpub.duke.edu

Sources of funding: National Institute of Mental Health, USA $\mathrm{p}=0.4$ ). Fluoxetine plus CBT significantly improved response rate compared with placebo or CBT alone $(p=0.001)$, but not compared with fluoxetine alone $(\mathrm{p}=0.11)$. Fluoxetine plus CBT produced the greatest reduction in suicidal thoughts $(\mathrm{p}=0.02)$.

\section{CONCLUSIONS}

Fluoxetine in combination with CBT is more effective than fluoxetine alone or CBT alone for improving symptoms of depression in adolescents with major depressive disorder

\section{NOTES}

Fluoxetine alone and placebo alone were given double blind, whereas CBT plus fluoxetine and CBT alone were given unblinded.

\section{Commentary}

Tr he Treatment for Adolescents with Depression Study (TADS) is notable for its rigorous design and timely clinical findings. Although acutely suicidal adolescents were necessarily excluded from the study, self harm, suicide attempts, and suicidal ideation were carefully monitored as they should be in clinical and research settings alike. Furthermore, the controversial use of placebo in depressed adolescents, coupled with careful monitoring, did not contribute to problems with safety or attrition and in fact resulted in some symptomatic benefit. Moreover, including a placebo group increased hypothesised group differences, therefore reducing required sample size, and consequently, the (potential) number of non-responders. '

Consider the timely nature of these results. In 2003 the UK's Drug Licensing Authority Committee on Safety of Medicines warned, "There is no, or insufficient, evidence from clinical trials that benefits outweigh the risks of side effects for sertraline (Lustral), citalopram (Cipramil), escitalopram (Cipralex) and fluvoxamine (Faverin). Fluoxetine, or Prozac, appears to have a positive balance of risks and benefits..."2 The US Food and Drug Administration (FDA) subsequently conducted an extensive review of all recent paediatric antidepressant $\mathrm{RCT}$ s involving over 4000 children. $^{3}{ }^{4}$ There were no fatalities in these trials. Most striking, however, only three of $15 \mathrm{RCT}$ s for MDD showed efficacy on the primary outcome. ${ }^{3}$ In fact, fluoxetine, which has a paediatric indication for depression, was the only antidepressant with more than one positive paediatric RCT supporting its use. ${ }^{56}$ Although suicidality (ideation or attempts) was uncommon in these trials, the risk:benefit ratio was disturbing. The FDA concluded that, although suicidality was rare, the risk was significantly higher in children receiving antidepressants relative to placebo. ${ }^{4}$ As a result, a black box warning regarding clinical worsening and suicide risk is now required on all antidepressants sold in the US, ${ }^{7}$ and the FDA has issued proposed guidance about using antidepressants in children and teenagers

This study is further evidence of the efficacy of fluoxetine for adolescents with depression, particularly when used in combination with CBT. It provides an important empirical basis for clinical decision making and is exemplary for the design of paediatric psychopharmacology RCTs. Andrew $\mathrm{C}$ Leon, $\mathrm{PhD}$

Professor of Biostatistics in Psychiatry and Professor of Public Health, Weill Medical College of Cornell University, NY, USA

Please visit http://www.ebmentalhealth.com/supplemental for reference list. 